\title{
THE PARAMETRIC GAUSS-GREEN THEOREM
}

\author{
M. ORTEL AND W. SCHNEIDER
}

\begin{abstract}
A strong parametric Gauss-Green theorem, for chains homologous to zero in a plane region, follows directly from an interchange in order of integrations.
\end{abstract}

1. Introduction. In this note we show that a strong parametric Gauss-Green theorem (Theorem 1 of $\S 3$ ) follows directly from an interchange of order of integration: the other steps in the proof are a short calculation in polar coordinates and a regularization, and these steps are obvious after the interchange. Only the definition and first properties of winding numbers (indices) appear (these are stated in §2). There are no topological constructions (which appear in all previous proofs).

Even though we emphasize simplicity of proof, it seems advisible to present the Gauss-Green theorem in the context of rectifiable chains and Lipschitzian integrands (defined in \$2). This level of generality does not change the method of proof; but now the calculation in polar coordinates requires references (especially Rademacher's theorem) which should be cited. This is done in Lemma 2 of $\S 2$. In the context of piecewise smooth chains and continuously differentiable integrands, the calculation is elementary and the short proof of the Gauss-Green theorem given in $\S 3$ is all that is required.

The strongest parametric Gauss-Green theorem (to our knowledge) is due to J. H. Michael [3], who is able to further weaken hypotheses on the integrand through a delicate topological construction. Also, in case our given rectifiable chain is composed of nonoverlapping simple closed curves, our theorem follows from the Gauss-Green theorem for sets of finite perimeter given in 4.5 .9 of H. Federer's Geometric measure theory [2]; the proof there involves much more than the proof given here.

The parametric Gauss-Green theorem motivates and implies the homology version of Cauchy's theorem, which has a well-known proof (in the elementary setting) due to J. D. Dixon [1]. That proof is short and avoids topological constructions; but the methods are based on properties of holomorphic functions and do not encompass the Gauss-Green theorem.

2. Definitions and references. We say $f$ is a Lipschitz function on $S$ (with Lipschitz constant $M$ ) if $S \subset \mathbf{C}$ (complex numbers) or $S \subset \mathbf{R}$ (real numbers), $f: S \rightarrow \mathbf{C}$, $M \in(0, \infty)$ and $|f(z)-f(w)| \leqslant M|z-w|$ whenever $z, w \in S$.

Received by the editors March 21, 1985 and, in revised form, October 7, 1985. 1980 Mathematics Subject Classification (1985 Revision). Primary 30E20, 30A99. 
We say $\gamma$ is a rectifiable 1-chain in $\Omega$ if $\gamma$ is a Lipschitz function on the union of a finite set of mutually disjoint compact nondegenerate intervals of $\mathbf{R}, \Omega$ is an open subset of $\mathbf{C}$, and image $\gamma \subset \Omega$.

Our terminology in measure theory is that of Federer (Chapter 2 of [2]). In particular, $\mathscr{L}^{1}$ denotes Lebesgue measure on $\mathbf{R}$ and $\mathscr{L}^{2}$ denotes Lebesgue measure on $\mathbf{R}^{2} \equiv \mathbf{C}$.

If $\gamma$ is a rectifiable 1-chain in $\Omega$ and $f: \Omega \rightarrow \mathbf{C}$ is continuous, we define

$$
\int_{\gamma} f(z) d z \equiv \int_{\text {domain } \gamma} f(\gamma(t)) \gamma^{\prime}(t) d \mathscr{L}^{1}(t) .
$$

By 2.5.17 and 2.9.21 of [2] the expression on the right is a complex number since $\gamma$ is absolutely continuous. This definition suffices because we may re-parametrize by arc length.

Suppose $\gamma$ is a rectifiable 1-chain in $\Omega$. We say $\gamma$ is closed if $L(\gamma)$ and $R(\gamma)$ denote the sets of left and right endpoints of domain $\gamma$ and if

$$
\operatorname{card}\{x \in L(\gamma): \gamma(x)=z\}=\operatorname{card}\{x \in R(\gamma): \gamma(x)=z\}
$$

whenever $z \in \mathbf{C}$. If $\gamma$ is closed and $z \in \mathbf{C}$, we denote

$$
\begin{array}{ll}
n(\gamma, z)=\frac{1}{2 \pi i} \int_{\gamma} \frac{d u}{u-z} & \text { if } z \notin \text { image } \gamma, \\
n(\gamma, z)=0 & \text { if } z \in \text { image } \gamma,
\end{array}
$$

and we call $n(\gamma, z)$ the index of $\gamma$ about $z$. We say $\gamma$ is homologous to zero modulo $\Omega$ if $\gamma$ is closed and $n(\gamma, z)=0$ whenever $z \in \mathbf{C}-\Omega$.

The proof of the following appears in textbooks on Complex Analysis and does not involve the Gauss-Green theorem

LEMMA 1. Suppose $\gamma$ is a closed rectifiable 1-chain in $\mathbf{C}$. Then the following are true:

(1.1) image $\gamma$ is a compact set;

(1.2) $n\left(\gamma, z_{1}\right)=n\left(\gamma, z_{2}\right)$ if $z_{1}$ and $z_{2}$ lie in the same component of $\mathbf{C}$-image $\gamma$;

(1.3) $n(\gamma, z)=0$ if $z$ lies in the unbounded component of C-image $\gamma$;

(1.4) $n(\gamma, z) \in\{0, \pm 1, \pm 2, \ldots\}$ all $z \in \mathbf{C}$.

If $f$ is a function and domain $f \cup$ image $f \subset \mathbf{C}$ we define the function

$$
\bar{\partial} f=\left\{\langle z, w\rangle: w=\frac{1}{2}\left(D_{1} f(z)+i D_{2} f(z)\right)\right\}
$$

where $D_{1}$ and $D_{2}$ denote partial derivatives.

LEMMA 2. Suppose $f$ is a Lipschitz function on C, $f$ has compact support, and $w \in$ C. Then

$$
\int_{\mathbf{C}} \frac{\bar{\partial} f(z)}{w-z} d \mathscr{L}^{2}(z)=\pi f(w) .
$$

Proof. Suppose $M$ is a Lipschitz constant for $f, \delta \in(0, \infty)$ and $f(z)=0$ when $|z-w| \geqslant \delta$. Define $h: \mathbf{C} \rightarrow \mathbf{C}$ by $h(r, \theta)=f\left(w+r e^{i \theta}\right)$ all $(r, \theta) \in \mathbf{R}^{2} \equiv \mathbf{C}$ and denote by $S(f)$ and $S(h)$ the sets of points in $\mathrm{C}$ at which $f$ and $h$ are differentiable (in the two variable sense). 
Now $h$ is a Lipschitz function on $\mathrm{C}$ (with constant $(1+\delta) M), h(r, \theta)=0$ if $|r| \geqslant \delta$ and $\theta \in \mathbf{R},|\bar{\partial} f(z)| \leqslant M$ when $z \in S(f)$, and

$$
\left|D_{1} h(r, \theta)\right|+\left|r^{-1} D_{2} h(r, \theta)\right| \leqslant 2 M
$$

whenever $(r, \theta) \in S(h)$ and $r \neq 0$. Moreover, if $(r, \theta) \in S(h)$ and $r \neq 0$, the chain rule implies $w+r e^{i \theta} \in S(f)$ and

$$
\bar{\partial} f\left(w+r e^{i \theta}\right)=\frac{e^{i \theta}}{2}\left(D_{1} h(r, \theta)+\frac{i}{r} D_{2} h(r, \theta)\right) .
$$

Since $f$ and $h$ are Lipschitz, Rademacher's theorem (3.1.6 of [2]) states

$$
\mathscr{L}^{2}(\mathbf{C}-S(f))=\mathscr{L}^{2}(\mathbf{C}-S(h))=0 ;
$$

thus (*) holds $\mathscr{L}^{2}$ almost everywhere and $D_{1} h, D_{2} h, \bar{\partial} f$ are $\mathscr{L}^{2}$ measurable functions (as limits by 2.3.2(6) of [2]). By measurability and the bounds on $|\bar{\partial} f|$ we conclude from the Transformation Theorem 3.2.5 of [2] that

$$
J(w) \equiv \int_{\mathbf{C}} \frac{\bar{\partial} f(z)}{w-z} d \mathscr{L}^{2}(z)=-\int_{(0, \delta] \times(0,2 \pi]} \bar{\partial} f\left(w+r e^{i \theta}\right) e^{-i \theta} d \mathscr{L}^{2}(r, \theta)
$$

and then by $(*)$ that

$$
J(w)=-\frac{1}{2} \int_{(0, \delta] \times(0,2 \pi]} D_{1} h(r, \theta)+\frac{i}{r} D_{2} h(r, \theta) d \mathscr{L}^{2}(r, \theta) .
$$

By the measurability and bounds on $\left|D_{1} h\right|$ and $\left|r^{-1} D_{2} h\right|$ we may apply Fubini's theorem (2.6.2 of [2]) to see

$$
\begin{aligned}
J(w)= & -\frac{1}{2} \int_{(0,2 \pi]} \int_{(0, \delta]} D_{1} h(r, \theta) d \mathscr{L}^{1}(r) d \mathscr{L}^{1}(\theta) \\
& -\frac{i}{2} \int_{(0, \delta]} r^{-1} \int_{(0,2 \pi]} D_{2} h(r, \theta) d \mathscr{L}^{1}(\theta) d \mathscr{L}^{1}(r) .
\end{aligned}
$$

Since $h$ is Lipschitz, the sections $r \rightarrow h(r, b)$ and $\theta \rightarrow h(a, \theta)$ are absolutely continuous (on any compact interval of $\mathbf{R}$ ) whenever $a, b \in \mathbf{R}$. It follows that

$$
\begin{aligned}
& \int_{(0, \delta]} D_{1} h(r, \theta) d \mathscr{L}^{1}(r)=h(\delta, \theta)-h(0, \theta)=0-f(w) \quad \text { if } \theta \in \mathbf{R}, \\
& \int_{(0,2 \pi]} D_{2} h(r, \theta) d \mathscr{L}^{1}(\theta)=h(r, 2 \pi)-h(r, 0)=0 \quad \text { if }-r \in \mathbf{R} .
\end{aligned}
$$

The proof is complete.

\section{The parametric Gauss-Green theorem.}

THEOREM 1. Suppose $\gamma$ is a closed rectifiable 1-chain in $\Omega, \gamma$ is homologous to zero modulo $\Omega$, and $f$ is a Lipschitz function on $\Omega$. Then

$$
\int_{\gamma} f(z) d z=2 i \int_{\Omega} n(\gamma, z) \bar{\partial} f(z) d \mathscr{L}^{2}(z) .
$$


REMARK. Theorem 1 implies that the area integral is a complex number even though $n(\gamma, \cdot)$ may be unbounded in $\Omega$.

Proof. Denote

$$
I \equiv\{z \in \mathbf{C}: n(\gamma, z) \neq 0\} \cup \text { image } \gamma .
$$

Now (1.1), (1.2), and (1.3) imply that $I$ is a closed and bounded subset of $\mathbf{C}$. Since $\gamma$ is homologous to zero modulo $\Omega$, we conclude $I$ is a compact subset of $\Omega$ and there is a Lipschitz function $\phi$ on $\mathbf{C}$ such that $\phi$ has compact support and $\phi(z)=f(z)$ for all $z$ in some open neighborhood of $I$. Then $\phi(z)=f(z)$ for all $z \in$ image $\gamma$ and $n(\gamma, z) \bar{\partial} \phi(z)=n(\gamma, z) \bar{\partial} f(z)$ for all $z \in \Omega$. Since $\gamma$ is homologous to zero modulo $\Omega, n(\gamma, z) \bar{\partial} \phi(z)=0$ when $z \in C-\Omega$. Therefore, the integrals in Theorem 1 are not changed if $\Omega$ and $f$ are replaced by $C$ and $\phi$. So it suffices to prove Theorem 1 in the case $\Omega=\mathbf{C}, f$ is Lipschitz on $\mathbf{C}$, and f has compact support.

With the extra assumptions in force set $T \equiv \operatorname{domain} \gamma$ and let $M$ denote a Lipschitz constant for $\gamma$. Now $\bar{\partial} f$ is $\mathscr{L}^{2}$ measurable, and $\gamma^{\prime}$ is $\mathscr{L}^{1} T$ (restriction) measurable, and the function $(t, z) \rightarrow(\gamma(t)-z)^{-1}$ is continuous on $T \times$ (C-image $\gamma$ ), and $\mathscr{L}^{2}$ (image $\left.\gamma\right)=0$ (easy to prove). So the function

$$
(t, z) \rightarrow \frac{\gamma^{\prime}(t) \bar{\partial} f(z)}{\gamma(t)-z} \text { is } \mathscr{L}^{1} \times \mathscr{L}^{2} T \times \mathbf{C} \text { measurable }
$$

and, by Fubini's theorem,

$$
\int_{T \times C} \frac{\left|\gamma^{\prime}(t) \bar{\partial} f(z)\right|}{|\gamma(t)-z|} d \mathscr{L}^{1} \times \mathscr{L}^{2}(t, z) \leqslant M \mathscr{L}^{1}(T) \sup _{w \in \mathbf{C}} \int_{\mathbf{C}} \frac{|\bar{\partial} f(z)|}{|w-z|} d \mathscr{L}^{2}(z)
$$

which is finite since $|\bar{\partial} f|$ is bounded with compact support. We conclude

$$
\int_{T} \gamma^{\prime}(t) \int_{\mathbf{C}} \frac{\bar{\partial} f(z)}{\gamma(t)-z} d \mathscr{L}^{2}(z) d \mathscr{L}^{1}(t)=\int_{\mathbf{C}} \bar{\partial} f(z) \int_{T} \frac{\gamma^{\prime}(t)}{\gamma(t)-z} d \mathscr{L}^{1}(t) d \mathscr{L}^{2}(z)
$$

and that the integrals are complex numbers. By Lemma 2

$$
\int_{\mathbf{C}} \frac{\bar{\partial} f(z)}{\gamma(t)-z} d \mathscr{L}^{2}(z)=\pi f(\gamma(t)) \text { for all } t \in T .
$$

Since $\mathscr{L}^{2}$ (image $\left.\gamma\right)=0$ we have

$$
\int_{T} \frac{\gamma^{\prime}(t)}{\gamma(t)-z} d \mathscr{L}^{1}(t)=2 \pi i n(\gamma, z)
$$

at $\mathscr{L}^{2}$ almost all $z \in \mathbf{C}$. The proof is complete.

\section{REFERENCES}

1. J. D. Dixon, A brief proof of Cauchy's integral theorem, Proc. Amer. Math. Soc. 29 (1971), 625-626.

2. H. Federer, Geometric measure theory, Springer, New York, 1969.

3. J. H. Michael, An approximation to a rectifiable plane curve, J. London Math. Soc. 30 (1955), 1-11.

Department of Mathematics, University of Hawail, Honolulu, Hawail 96822

Department of Mathematics, Carleton University, Ottawa, Ontario K1S 5B6, Canada 